\title{
Definition of Vascular Territories on Myocardial Perfusion Images by Integration with True Coronary Anatomy: A Hybrid PET/CT Analysis
}

Mehrbod S. Javadi, Riikka Lautamäki, Jennifer Merrill, Corina Voicu, William Epley, Gerald McBride, and Frank M. Bengel

Division of Nuclear Medicine, Russell H. Morgan Department of Radiology, Johns Hopkins University, Baltimore, Maryland

For interpretation of myocardial perfusion studies, tissue segments are usually assigned to coronary vascular territories based on general assumptions about the most frequent vascular distribution pattern. These assumptions may be inaccurate because of interindividual variability of coronary anatomy. This limitation may be overcome by hybrid imaging through the individual integration of coronary anatomy with myocardial tissue regions. Methods: We studied 71 consecutive patients who underwent ${ }^{82} \mathrm{Rb}$ perfusion $\mathrm{PET} / \mathrm{CT}$, including CT angiography, for work-up of coronary artery disease on a 64-slice PET/CT scanner. Coronary vessels as defined by CT were assigned to each of 17 myocardial segments for PET analysis using fusion images. Reassigned segmental maps were compared with standard assignment as proposed by the American Heart Association model, without knowledge of individual anatomy. The validity of segmental assignment was tested in 6 dogs by comparison of $\mathrm{PET} / \mathrm{CT}$ with ex vivo dye staining of coronary territories. Results: Dog studies showed excellent agreement between PET/CTdefined segments and ex vivo-stained territories $(\kappa, 0.80)$. In patients, $72 \%$ (51/71) demonstrated differences from the standard assignment in at least 1 myocardial segment; 112 of 1,207 segments were reassigned to nonstandard vascular territories. Most frequently, standard right coronary segments were reassigned to the left circumflex territory (39\% of reassigned segments), standard circumflex segments were reassigned to the left anterior descending territory (30\%), and standard left anterior descending segments were reassigned to either circumflex or right coronary (12\% and $11 \%$, respectively). In 27 studies with a myocardial perfusion defect, relative uptake in the vascular territory with the defect was significantly lower after CT-based reassignment and was higher in remote territories, resulting in better separation (ratio of defect to remote, $0.75 \pm 0.13$ vs. $0.81 \pm 0.12$ before reassignment; $P=0.0014)$. Conclusion: Standard assumptions about vascular territory distribution in myocardial perfusion analysis are frequently inaccurate because of morphologic variability of the coronary tree. If hybrid imaging has been used to study coronary anatomy and myo-

Received Jun. 17, 2009; revision accepted Oct. 30, 2009.

For correspondence or reprints contact: Frank M. Bengel, Division of Nuclear Medicine, Russell H. Morgan Department of Radiology, Johns Hopkins University, 601 N. Caroline St., JHOC 3225, Baltimore, MD 21287.

E-mail: fbengel1@jhmi.edu

COPYRIGHT @ 2010 by the Society of Nuclear Medicine, Inc. cardial tissue perfusion, then localization of perfusion abnormalities should be based on CT-derived anatomy. This may bring about more accurate assignment to culprit vessels and thus improved guidance and monitoring of targeted therapy.

Key Words: hybrid imaging; PET/CT; CT coronary angiography; vascular territories

J Nucl Med 2010; 51:198-203

DOI: 10.2967/jnumed.109.067488

$\mathrm{F}$

or structured and standardized visual analysis of noninvasive myocardial imaging studies, a 17-segment model of the left ventricle has been established (1). Although it is well recognized that the anatomy of the coronary tree can be variable, this 17-segment model proposes a fixed assignment of segments to 1 of the 3 major vascular territories: the territory of the left anterior descending coronary artery (LAD), left circumflex (LCX) coronary artery, or right coronary artery (RCA).

Myocardial perfusion imaging is typically used as a gatekeeper before invasive work-up, and the detection of ischemia is used to guide targeted intervention (2). Misassignment of myocardial segments because of a deviation of true coronary anatomy from the standard model could alter the identification of the involved coronary vessel or the location of the culprit lesion.

The advent of noninvasive coronary angiography and hybrid imaging may provide a solution to this problem. Hybrid SPECT/CT and PET/CT allow for the integrated analysis of coronary anatomy and myocardial perfusion or function (3). Several studies have suggested that morphology and function are complementary for the diagnosis and prognosis of coronary artery disease (CAD) (4-9). Additionally, fusion of the coronary tree with myocardial imaging facilitates the assignment of perfusion defects to a respective culprit vessel $(10,11)$.

An individual, accurate assignment of myocardial segments to coronary territories may be another advantage of 
a combined functional and morphologic imaging approach to $\mathrm{CAD}$. We speculated that the standard assignment of vascular territories as defined by the American Heart Association (AHA) segmental model would frequently change when the true coronary anatomy is known, and we tested this hypothesis using state-of-the-art hybrid PET/CT methodology.

\section{MATERIALS AND METHODS}

\section{Patients}

Seventy-one consecutive patients (41 women, 30 men; mean age $\pm \mathrm{SD}, 56 \pm 14 \mathrm{y}$; mean body mass index $\pm \mathrm{SD}, 32 \pm 8$ ) with an intermediate pretest likelihood of CAD were referred for morphologic and functional evaluation of chest pain. Coronary morphology and myocardial perfusion were assessed using a 64-slice Discovery Rx VCT PET/CT scanner (GE Healthcare). Patients with arrhythmia, contraindications to radiographic contrast, or contraindications to dipyridamole stress were excluded. Retrospective analysis for this project was granted exempt status by the Johns Hopkins Institutional Review Board.

\section{PET/CT Acquisition Protocol}

PET. All patients fasted for more than $4 \mathrm{~h}$ before testing. Abstinence from caffeine for more than $12 \mathrm{~h}$ and from theophylline-containing medications or vasodilators for more than $24 \mathrm{~h}$ was required. Perfusion PET constituted the first part of the PET/ $\mathrm{CT}$ session. In all patients, an appropriate history and informed consent were secured, and a large intravenous line ( $>20$ gauge) was placed in an antecubital vein before imaging. Individuals were positioned with the help of a CT topogram, and a low-dose CT scan $(120 \mathrm{kV}, 50-100 \mathrm{~mA})$ for attenuation correction of PET emission data was acquired during shallow breathing. Then, ${ }^{82} \mathrm{Rb}-$ chloride $(1,480-1,850 \mathrm{MBq}$ [40-50 $\mathrm{mCi}])$ was infused intravenously from a strontium/rubidium generator as a slow bolus over $30 \mathrm{~s}$, and a list-mode 2-dimensional PET image was acquired over $8 \mathrm{~min}$. After the rest acquisition, infusion of dipyridamole was started $(0.56 \mathrm{mg} / \mathrm{kg}, 4 \mathrm{~min})$, and a second dose of ${ }^{82} \mathrm{Rb}$-chloride $(1,480-1,850 \mathrm{MBq}[40-50 \mathrm{mCi}])$ was infused at $4 \mathrm{~min}$ after the end of dipyridamole infusion, followed by an 8-min list-mode acquisition. The rest and stress PET data were checked for accurate alignment with the low-dose CT data for attenuation correction, and software-based realignment was performed if necessary (12). List-mode data were resampled to static images for perfusion analysis (90-s prescan delay, individually increased in cases of slow ${ }^{82} \mathrm{Rb}$ blood clearance). To counteract the effects of dipyridamole, aminophylline (100-150 mg) was given at the end of the PET scan.

CT Angiography (CTA). The CT acquisition protocol was initiated immediately after the end of the PET acquisition. To reduce heart rate, patients were premedicated with oral metoprolol (50-100 $\mathrm{mg}, 1 \mathrm{~h}$ before the CT scan). Once heart rate dropped below the target of $65 / \mathrm{min}$, CT acquisition was started with a scout scan to determine start and end positions. To determine the optimal timing of contrast delivery, we gave a test bolus injection of $20 \mathrm{~mL}$ of intravenous contrast (Visipaque; GE Healthcare) ( $5 \mathrm{~mL} / \mathrm{s}$, followed by $20 \mathrm{~mL}$ of saline), with repeated imaging of the aortic root afterward. Then, $0.2 \mathrm{~g}$ of nitroglycerin was given sublingually for coronary vasodilation, and CTA was performed with $70 \mathrm{~mL}$ of contrast injected at $5 \mathrm{~mL} / \mathrm{s}$, followed by a $50-\mathrm{mL}$ saline chaser at the same speed. In the first 37 patients, a helical acquisition protocol was used with the following settings: pitch, 0.2-0.24 (dependent on heart rate); $120 \mathrm{kV}$; milliamperage modulated (maximum of 600-800 MA during the 60\%-85\% phase, minimum of $400 \mathrm{~mA}$ ); rotation time, $0.35 \mathrm{~s}$; retrospective gating; and segmented reconstruction of multiple phases throughout the R-R cycle. In the subsequent 34 patients, a new low-dose protocol (13) was applied using step-and-shoot mode, to cover the area from below the tracheal bifurcation to the diaphragm (rotation time, $0.35 \mathrm{~s}$; prospective gating; center of acquisition at $75 \%$ of the R-R interval; 3-4 scan blocks, 1 block acquired every 2 heartbeats; $z$-axis coverage of $40 \mathrm{~mm}$ per block; increment, $35 \mathrm{~mm}$; slice thickness, $0.625 \mathrm{~mm} ; 120 \mathrm{keV}$; 600-800 $\mathrm{mA}$, dependent on weight).

\section{Data Analysis}

Coronary Anatomy. The single-best phase of helical CTA or the single phase of step-and-shoot CTA was used for the analysis of coronary anatomy and assignment of myocardial vascular supply. Three-dimensional volumetric rendering was used to determine gross coronary anatomy. The coronary tree was assessed for vessel dominance (based on the supply of the posterior descending artery), the presence of large side branches, and other nonstandard features (Fig. 1).

Assignment of Myocardial Segments to Coronary Vessels. From CTA datasets, maximum-intensity-projection images perpendicular to the cardiac long axis were created for direct comparison with the AHA segmental model used for myocardial perfusion analysis (Fig. 2). The territory of 1 of 3 coronary arteries (LAD,

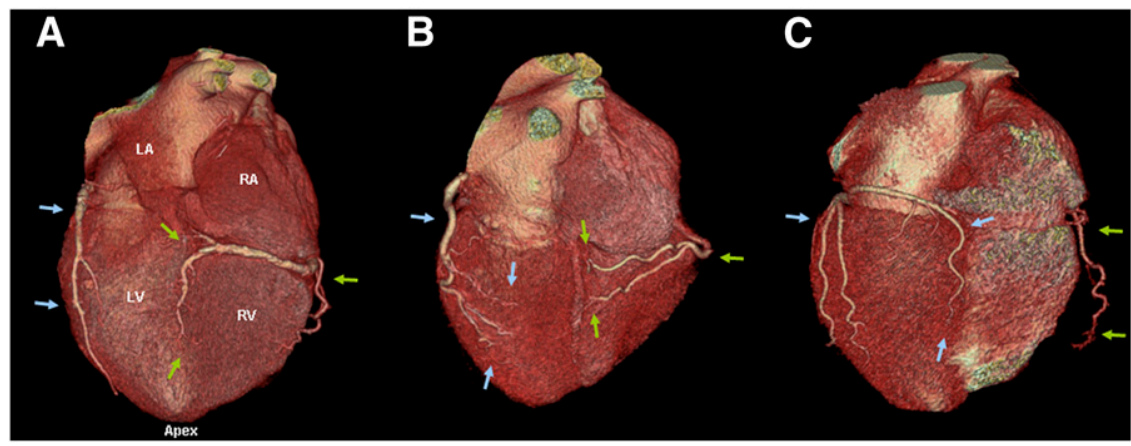

FIGURE 1. Three-dimensional, volume-rendered inferior and posterior CT angiographic images showing different coronary anatomy variants. (A) Standard, right-dominant circulation. Right coronary artery (green arrows) supplies posterior descending branch. Left circumflex (blue arrows) supplies only inferolateral left ventricular myocardium. (B) Codominant circulation. No clear posterior descending artery is observed. Inferior myocardium is supplied by both right and left circumflex arteries. (C) Left-dominant circulation. Left circumflex supplies posterior descending branch. Right coronary artery supplies only right ventricular myocardium. $L A=$ left atrium; RA = right atrium; LV = left ventricle; RV = right ventricle. 


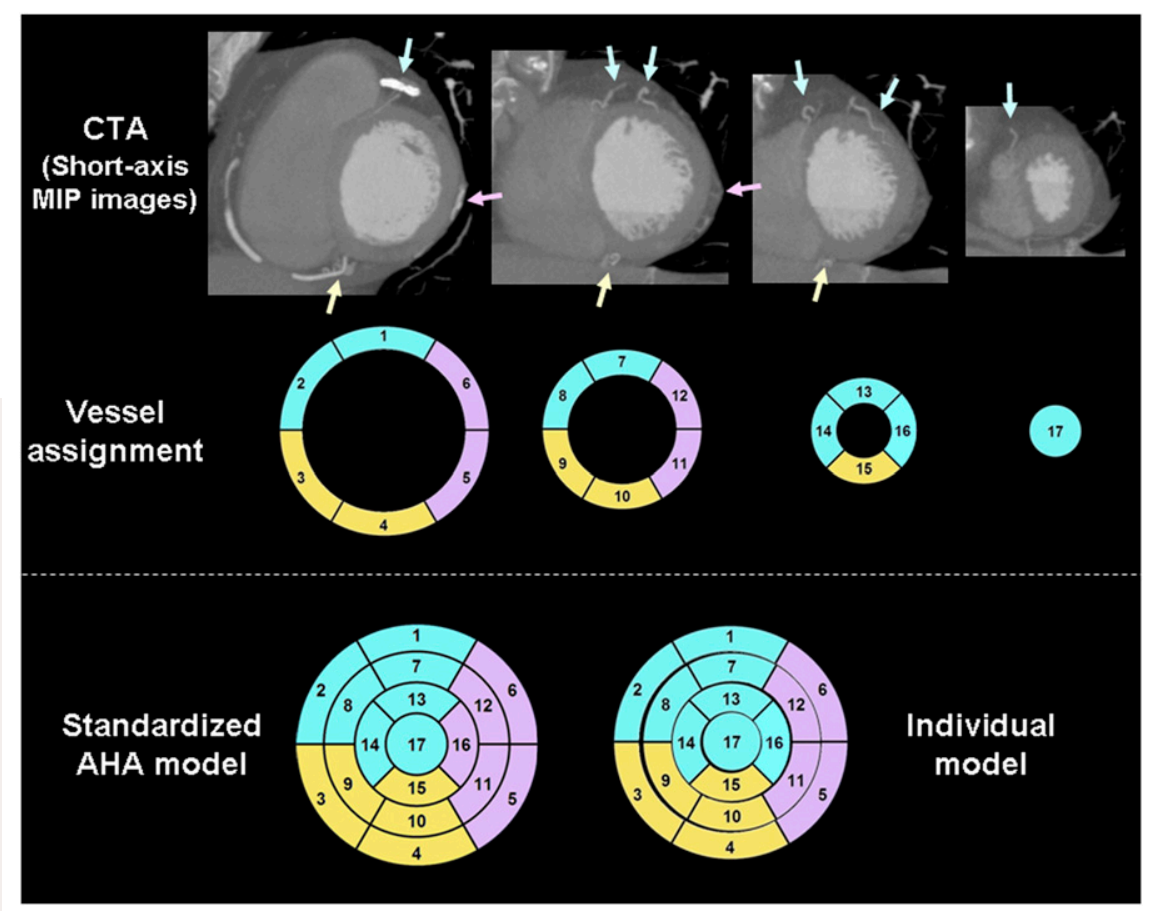

FIGURE 2. CT-based individual assignment of myocardial segments to vascular territories. Short-axis maximum-intensity-projection (MIP) images of CTA are reviewed for localization of coronary arteries (top). Each of 17 myocardial segments is assigned to closest coronary artery (middle; LAD in blue, LCX in pink, and RCA in yellow). Bottom shows standard AHA model on left and model after individual reassignment on right. In this case, segment 16 (distal lateral wall) was reassigned to LAD, which supplied diagonal branch.

LCX, or RCA) was assigned to each of 17 myocardial segments (1). A ramus intermedius was generally treated like a large-caliber diagonal branch and was assigned to the LAD territory. When a segment was deemed to have a dual supply, both vascular territories were assigned and the segment was considered to have a nonstandard supply. This assignment was performed by 2 experienced readers blinded to each other and the PET images. Discrepancy was resolved by consensus after the initial read.

Effect on Semiquantitative Tracer Uptake. From studies with a regional perfusion defect on visual analysis, polar maps of static myocardial tracer uptake were created using volumetric sampling (14). Maps were normalized to the individual left ventricular maximum. Percentage uptake in vascular territories was compared using both the standardized 17 segment model and the CT-defined individualized segmental model, for territories with and without defect.

\section{Experimental Validation of Segmental Assignment}

Data from 6 mongrel dogs with experimental coronary stenosis were available from a previous study (15) and were reanalyzed for the purpose of this study. Animals had undergone PET/CT, including CTA, as previously described (15). After imaging, 1 coronary vessel (LAD, $n=3$; LCX, $n=3$ ) was totally occluded and monastral blue $(0.5 \mathrm{~mL} / \mathrm{kg})$ was injected into the left atrium to stain myocardium remote to the stenotic vessel territory. The animal was then euthanized, the heart was excised, the left ventricle was surgically isolated, macroscopic short-axis slices of approximately $1-\mathrm{cm}$ thickness were created, and photographs of the dye-stained slices were obtained.

Because dogs are characterized by a left-dominant circulation, the human segmental model does not apply. But to validate segmental assignment, CT data were used to assign 17 segments to either LAD or LCX, using the same approach as that applied in humans. These segments were then compared with the presence or absence of dye staining ex vivo (Fig. 3).

\section{Statistical Analysis}

Results are reported as mean $\pm \mathrm{SD}$. Statistical analysis was performed with MedCalc software. $\kappa$-statistics were calculated to determine interobserver agreement for coronary tree analysis and assignment of myocardial segments to vascular territories and to determine agreement between in vivo and ex vivo segmental distribution in dogs. PET parameters in vascular territories before and after CT-based segmental reassignment were compared by 2-tailed paired Student $t$ test. A $P$ value less than 0.05 was considered statistically significant.

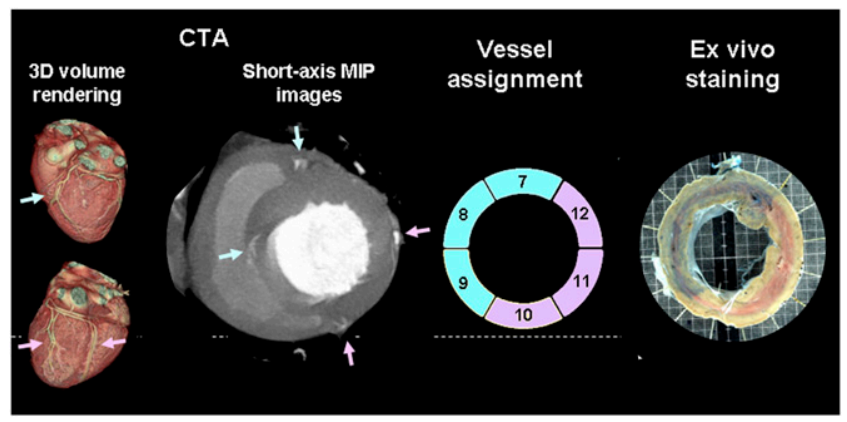

FIGURE 3. Experimental validation of CT-based visual assignment of myocardial segments. Dog model was used, which generally shows left-dominant distribution, as shown on 3-dimensional volume-rendered surface images of CT (left). Representative midventricular short-axis maximumintensity-projection (MIP) slice of CTA in dog is shown (middle left), along with respective assignment of myocardial segments to vascular territories (middle right; blue and pink arrows indicate LAD and LCX). Ex vivo images of dyestained heart (right) show excellent agreement with true vessel distribution (nonoccluded LAD is stained in blue, and occluded LCX is not stained). 


\section{RESULTS}

The analysis of gross coronary morphology showed the standard pattern of right dominance in $80 \%$ (57/71) of patients. Left dominance was found in 11 (16\%), codominance in $2(3 \%)$, and a single coronary artery in 1 individual (because of a rare condition, this subject was removed from further segmental analysis). A trifurcation of the left coronary artery with a ramus intermedius was identified in 15 patients $(21 \%)$, and 7 patients (10\%) were judged to have a balanced contribution of LAD diagonal branches and LCX marginal branches to supply the lateral wall. Coronary atherosclerosis was reported in 50 of 71 patients (71\%; judged to be nonobstructive in 39 and obstructive in 11; no total occlusions).

For individual CT-based assignment of the vascular supply of myocardial segments, interobserver agreement was good, with a $\kappa$-value of 0.64 for nonstandard LAD segments, 0.79 for nonstandard LCX segments, and 0.66 for nonstandard RCA segments. The validity of CT-based segmental assignment was supported by excellent agreement with ex vivo staining of coronary territories in dogs, in which the assignment was identical in $91 \%$ of segments (к-value of 0.80 ).

Although most patients had the typical right-dominant pattern, at least 1 segment differed from the standard AHA model in $72 \%$ of patients $(51 / 71)$. On average, $2.0 \pm 1.7$ myocardial segments per patient demonstrated a nonstandard vascular supply. One hundred twelve of 1,207 included segments were assigned to nonstandard territories based on segmental remapping. Only 9 segments were judged to have a balanced, dual supply by 2 vascular territories. Figure 4 summarizes the changes. The reassignment of segments occurred most frequently in the inferior wall (RCA territory), although all territories were affected.

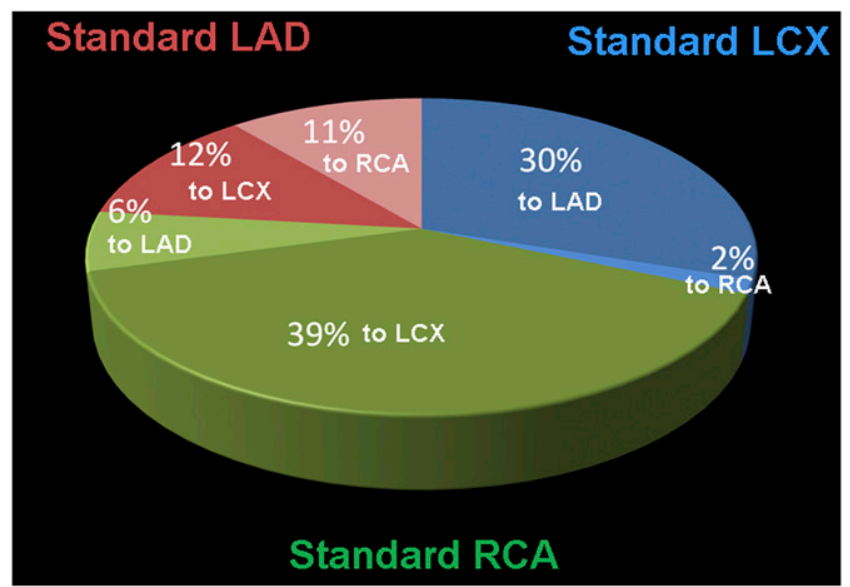

FIGURE 4. Distribution of 112 segments, which were reassigned after individual review of CT. Original segmental assignment to vascular territories is shown by color, along with percentage of overall segments that were reassigned and their new vascular territory.
No segment in patients had to be rejected from vascular assignment because the supplying vessel could not be seen.

Twenty-seven studies showed perfusion defects on visual analysis ( 5 of those in the lateral wall). In those 27 studies, CT-based reassignment of segments resulted in a significantly lower average uptake in the territory supplied by the culprit vessel $(55 \% \pm 11 \%$ of maximum vs. $58 \% \pm 10 \%$ using the standard segmental model; $P=0.0016)$ and significantly higher uptake in vascular territories without perfusion defects $(74 \% \pm 7 \%$ vs. $71 \% \pm 8 \%$ before reassignment; $P=0.0086$ ). Overall, the reassignment of segments resulted in a better separation of the defect territory from normal territories (ratio of defect territory to remote territories, $0.75 \pm 0.13$ vs. $0.81 \pm 0.12$ before reassignment; $P=0.0014$ ). Figure 5 shows an example of the effect of individual CT-based segmental reassignment on semiquantitative perfusion in hypoperfused territories versus normal territories.

\section{DISCUSSION}

Our integrated PET/CT analysis reveals that the standard assignment of myocardial segments to vascular territories proposed by the AHA 17-segmental model is frequently inaccurate. Our analysis also shows that the individual assignment of vascular supply by visual analysis of contrast-enhanced CTA is feasible, reproducible, and valid. A large fraction of individuals in our study (72\%) showed deviation of at least 1 segment when myocardial perfusion was integrated with true, CTA-derived coronary morphology. Accordingly, semiquantitative perfusion was lower in vascular territories with a perfusion defect and higher in those without when individual CT-driven assignment of segments was used. This result suggests that ischemia and infarcts in the territory of a culprit vessel are better distinguished from remote territories. The resulting improvement of precision may have implications for clinical decision making and for monitoring of targeted interventions.

Our study highlights that individual anatomy-driven assignment of myocardial segments for perfusion studies may be another advantage of integrated radionuclide and CT of the heart. Several prior studies already suggested a variety of benefits from combined imaging of physiology and morphology: First, in hybrid systems, the CT may be used for attenuation correction of nuclear data, to improve accuracy and accelerate acquisition protocols $(12,16,17)$. Second, nuclear and CT data seem to be complementary for diagnostic $(4-8)$ and prognostic $(9,18)$ work-ups of CAD. And finally, fusion imaging may be helpful in determining the hemodynamic relevance of coronary artery stenoses $(11,19)$. Taken together, all these studies support a combined approach of using noninvasive angiography along with perfusion studies.

Using separately acquired invasive angiography and functional noninvasive imaging, other recent studies have 
FIGURE 5. Polar map of stress myocardial perfusion from ${ }^{82} \mathrm{Rb} \mathrm{PET}$, showing perfusion defects in distal anterior wall and apex and in basal inferior wall. CTA shows codominant circulation with obstructive atherosclerosis of mid LAD and mid LCX artery. With standard AHA segment assignment (top right), inferior wall defect contributes to reduced perfusion mostly in RCA territory. After CT-based individual assignment (bottom right), inferior wall defect contributes mostly to LCX territory. Average perfusion tracer uptake per vascular territory changes significantly for RCA and LCX (right).

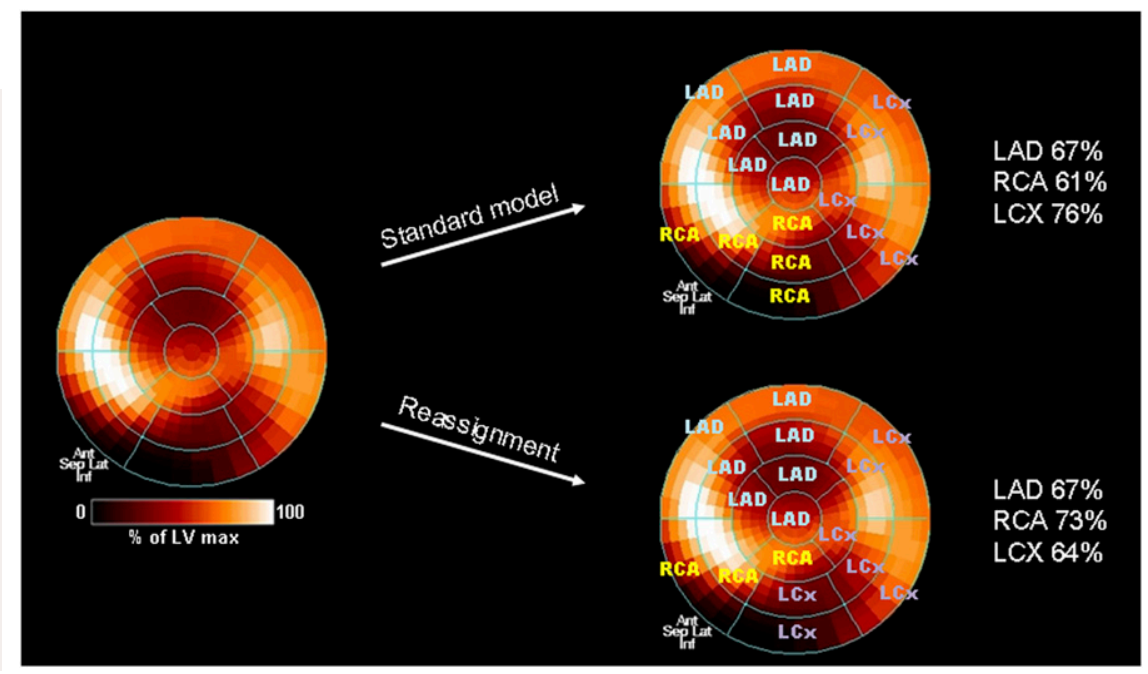

suggested that a generalized application of the AHA segmental model for vascular territory assignment may have limitations. Pereztol-Valdes et al. studied 50 patients scheduled for percutaneous intervention and injected ${ }^{99 \mathrm{~m}} \mathrm{Tc}$-sestamibi during balloon inflation (20). The subsequent comparison of defects on SPECT images with the standard segmental assignment by the AHA model showed that only 9 of the 17 segments could be unanimously assigned to a single coronary artery in all patients. Eight of those were segments from the LAD territory, and 1 was from the LCX territory. All other segments showed a wide variety of vascular supply. More recently, Ortiz-Perez et al. compared delayed segmental gadolinium hyperenhancement at MRI with invasive angiography in 93 patients after myocardial infarction (21). They showed discordance between hyperenhancing segments and empirically assigned coronary distribution according to the AHA model, which was most pronounced in the RCA and LCX regions. Concordantly with these prior studies, the inferior wall demonstrated the largest variability of vascular supply in our study, with nonstandard assignments to both the LAD and the LCX territories. This was usually a result of either left-dominant or codominant circulations. Several patients also demonstrated a large-caliber LAD, which extended to supply the distal inferior wall of the heart, which is normally assigned to distal branches of the RCA. Notably, however, there was also variability in the LAD or LCX border zone, mostly related to the presence of larger LAD diagonal branches, a ramus intermedius, or larger LCX obtuse marginal branches. This variability resulted in the nonstandard reassignment of segments in the anterior and lateral walls.

Dedicated software for a more automated fusion of CTA and myocardial perfusion studies was not included in our analysis. Some software approaches have been introduced, initially for the fusion of invasive angiography and SPECT (22) and more recently also for the fusion of noninvasive angiography and nuclear studies $(11,19)$. Individual assign- ment of myocardial segments to vascular territories is not yet a routine feature of this software, but our results suggest that such an implementation may be useful. Some potential limitations of CT-based assignment of vascular territories should be emphasized. First, myocardial flow patterns in the case of occluded and collateralized vessels may not necessarily match the angiogram. In patients with advanced disease, the situation may thus be more complex. Second, diffuse disease and occlusion and a dense supply caused by competing vessels such as LAD diagonal and LCX marginal branches in the lateral wall may complicate individual vessel assignment and reduce accuracy. In our study, however, no segment needed to be rejected from vascular assignment, and less than $1 \%$ of segments could not be assigned to a single dominant supplying vessel, suggesting that quality of vessel visualization on CT is rarely a limiting factor. In this regard, it should be considered that pure visualization of the course of a vessel is less demanding than is assessment of the vessel wall for plaque and stenosis, which is the dominant purpose of angiography. Additionally, calcified plaque may allow for tracking of vessels, even in the case of poor contrast opacification.

Although additive radiation exposure has been a concern for the widespread combination of CTA and radionuclide myocardial perfusion studies, new approaches to reduce exposure time for CT (13) and injected dose for SPECT and PET $(23,24)$ are expected to reduce effective dose significantly. Those, together with the increasing evidence of clinical usefulness, will likely result in a broader implementation of integrated functional and anatomic imaging in the future. There are several clinical scenarios in which an individual assignment of myocardial segments may be valuable. First, it may help in distinguishing single- and multivessel disease. Second, it may also help in guiding targeted revascularization, especially in the setting of multivessel disease. Third, it may help to more accurately assess the severity of ischemic damage in a given vascular territory. The accurate assessment of ischemic damage may 
not only facilitate the decision for or against targeted therapy but also improve the accuracy of follow-up studies to determine the effect of any therapy on ischemic burden. None of those scenarios was tested specifically in our study, but our results should be seen as a stimulus for subsequent larger trials.

\section{CONCLUSION}

The integration of myocardial perfusion imaging with concomitant CTA provides a more accurate assignment of vascular distribution. Thus, if hybrid imaging has been used to study coronary anatomy and myocardial tissue in an individual, then perfusion defect localization should be based on CT-defined individual anatomy rather than the general assumptions of the AHA model. Localizing perfusion defects on this basis will aid in the identification of culprit vessels for improved localization before targeted therapy.

\section{REFERENCES}

1. Cerqueira MD, Weissman NJ, Dilsizian V, et al. Standardized myocardial segmentation and nomenclature for tomographic imaging of the heart: a statement for healthcare professionals from the Cardiac Imaging Committee of the Council on Clinical Cardiology of the American Heart Association. Circulation. 2002;105:539-542.

2. Brindis RG, Douglas PS, Hendel RC, et al. ACCF/ASNC appropriateness criteria for single-photon emission computed tomography myocardial perfusion imaging (SPECT MPI): a report of the American College of Cardiology Foundation Quality Strategic Directions Committee Appropriateness Criteria Working Group and the American Society of Nuclear Cardiology endorsed by the American Heart Association. J Am Coll Cardiol. 2005;46:1587-1605.

3. Di Carli MF, Hachamovitch R. New technology for noninvasive evaluation of coronary artery disease. Circulation. 2007;115:1464-1480.

4. Di Carli MF, Dorbala S, Curillova Z, et al. Relationship between CT coronary angiography and stress perfusion imaging in patients with suspected ischemic heart disease assessed by integrated PET-CT imaging. J Nucl Cardiol. 2007;14:799-809.

5. Gaemperli O, Schepis T, Koepfli P, et al. Accuracy of 64-slice CT angiography for the detection of functionally relevant coronary stenoses as assessed with myocardial perfusion SPECT. Eur J Nucl Med Mol Imaging. 2007;34:1162-1171.

6. Hacker M, Jakobs T, Matthiesen F, et al. Comparison of spiral multidetector CT angiography and myocardial perfusion imaging in the noninvasive detection of functionally relevant coronary artery lesions: first clinical experiences. $\mathrm{J} \mathrm{Nucl}$ Med. 2005;46:1294-1300.

7. Rispler S, Keidar Z, Ghersin E, et al. Integrated single-photon emission computed tomography and computed tomography coronary angiography for the assessment of hemodynamically significant coronary artery lesions. J Am Coll Cardiol. 2007;49:1059-1067.
8. Schuijf JD, Wijns W, Jukema JW, et al. Relationship between noninvasive coronary angiography with multi-slice computed tomography and myocardial perfusion imaging. J Am Coll Cardiol. 2006;48:2508-2514.

9. van Werkhoven JM, Schuijf JD, Gaemperli O, et al. Prognostic value of multislice computed tomography and gated single-photon emission computed tomography in patients with suspected coronary artery disease. J Am Coll Cardiol. 2009;53:623-632.

10. Gaemperli O, Schepis T, Kalff V, et al. Validation of a new cardiac image fusion software for three-dimensional integration of myocardial perfusion SPECT and stand-alone 64-slice CT angiography. Eur J Nucl Med Mol Imaging. 2007;34: 1097-1106.

11. Gaemperli O, Schepis T, Valenta I, et al. Cardiac image fusion from stand-alone SPECT and CT: clinical experience. J Nucl Med. 2007;48:696-703.

12. Lautamaki R, Brown TL, Merrill J, Bengel FM. CT-based attenuation correction in ${ }^{82} \mathrm{Rb}$-myocardial perfusion PET-CT: incidence of misalignment and effect on regional tracer distribution. Eur J Nucl Med Mol Imaging. 2008;35:305-310.

13. Javadi M, Mahesh M, McBride G, et al. Lowering radiation dose for integrated assessment of coronary morphology and physiology: first experience with stepand-shoot CT angiography in a rubidium 82 PET-CT protocol. J Nucl Cardiol. 2008;15:783-790.

14. Nekolla SG, Miethaner C, Nguyen N, Ziegler SI, Schwaiger M. Reproducibility of polar map generation and assessment of defect severity and extent assessment in myocardial perfusion imaging using positron emission tomography. Eur $J$ Nucl Med. 1998;25:1313-1321.

15. Lautamaki R, George RT, Kitagawa K, et al. Rubidium-82 PET-CT for quantitative assessment of myocardial blood flow: validation in a canine model of coronary artery stenosis. Eur J Nucl Med Mol Imaging. 2009;36:576-586.

16. Goetze S, Brown TL, Lavely WC, Zhang Z, Bengel FM. Attenuation correction in myocardial perfusion SPECT/CT: effects of misregistration and value of reregistration. J Nucl Med. 2007;48:1090-1095.

17. Schepis T, Gaemperli O, Koepfli P, et al. Use of coronary calcium score scans from stand-alone multislice computed tomography for attenuation correction of myocardial perfusion SPECT. Eur J Nucl Med Mol Imaging. 2007;34:11-19.

18. Schenker MP, Dorbala S, Hong EC, et al. Interrelation of coronary calcification, myocardial ischemia, and outcomes in patients with intermediate likelihood of coronary artery disease: a combined positron emission tomography/computed tomography study. Circulation. 2008;117:1693-1700.

19. Santana CA, Garcia EV, Faber TL, et al. Diagnostic performance of fusion of myocardial perfusion imaging (MPI) and computed tomography coronary angiography. J Nucl Cardiol. 2009;16:201-211.

20. Pereztol-Valdes O, Candell-Riera J, Santana-Boado C, et al. Correspondence between left ventricular 17 myocardial segments and coronary arteries. Eur Heart J. 2005;26:2637-2643.

21. Ortiz-Perez JT, Rodriguez J, Meyers SN, Lee DC, Davidson C, Wu E. Correspondence between the 17-segment model and coronary arterial anatomy using contrast-enhanced cardiac magnetic resonance imaging. JACC Cardiovasc Imaging. 2008;1:282-293.

22. Faber TL, Santana CA, Garcia EV, et al. Three-dimensional fusion of coronary arteries with myocardial perfusion distributions: clinical validation. J Nucl Med. 2004;45:745-753.

23. Depuey EG. New software methods to cope with reduced counting statistics: shorter SPECT acquisitions and many more possibilities. J Nucl Cardiol. 2009;16:335-338.

24. Knesaurek K, Machac J, Krynyckyi BR, Almeida OD. Comparison of 2-dimensional and 3-dimensional ${ }^{82} \mathrm{Rb}$ myocardial perfusion PET imaging. J Nucl Med. 2003;44:1350-1356. 